\title{
Heritability and risks associated with early onset hypertension: multigenerational, prospective analysis in the Framingham Heart Study
}

\author{
Teemu J Niiranen, ${ }^{1}$ Elizabeth L McCabe, ${ }^{2}$ Martin G Larson, ${ }^{1,2}$ Mir Henglin, ${ }^{3}$ Neal K Lakdawala, ${ }^{3}$ \\ Ramachandran S Vasan, ${ }^{1,4,5,6}$ Susan Cheng ${ }^{1,3}$
}

$\overline{{ }^{1} \text { National Heart, Blood and Lung }}$

Institute's and Boston

University's Framingham Heart

Study, Framingham, MA 01702,

USA

${ }^{2}$ Department of Biostatistics,

Boston University School of

Public Health, Boston, MA, USA

${ }^{3}$ Division of Cardiovascular

Medicine, Department of

Medicine, Brigham and

Women's Hospital, Boston,

MA, USA

${ }^{4}$ Section of Preventive Medicine,

Department of Medicine,

Boston University School of

Medicine, Boston, MA, USA

5Section of Cardiology,

Department of Medicine,

Boston University School of

Medicine, Boston, MA, USA

${ }^{6}$ Department of Epidemiology,

Boston University School of

Public Health, Boston, MA, USA

Correspondence to: $\mathrm{TJ}$

Niiranen teemu.niiranen@thl.fi

Additional material is published

online only. To view please visit

the journal online.

Cite this as: BMJ 2017;357:j1949

http://dx.doi.org/10.1136/bmj.j1949

Accepted: 17 April 2017

\section{ABSTRACT}

OBJECTIVE

To determine the role of early onset versus late onset hypertension as a risk factor for hypertension in offspring and cardiovascular death.

DESIGN

Multigenerational, prospective cohort study.

SETTING

Framingham Heart Study.

\section{PARTICIPANTS}

Two generations of community dwelling participants with blood pressure measurements performed at serial examinations spanning six decades: 3614 first generation participants with mortality data and 1635 initially non-hypertensive second generation participants with data available on parental blood pressure.

\section{MAIN OUTCOME MEASURES}

The main outcome measures were relation of parental early onset hypertension (age $<55$ years) with incidence of hypertension in offspring, using regression analyses, and relation of age at hypertension onset with cause specific mortality using a case (cardiovascular death) versus control (noncardiovascular death) design.

RESULTS

In second generation participants, having one or both parents with late onset hypertension did not increase the risk of hypertension compared with having parents with no hypertension; by contrast, the hazard ratios of hypertension were 2.0 (95\% confidence interval 1.2 to 3.5) and 3.5 (1.9 to 6.1) in participants with one and both parents with early onset hypertension, respectively. In first generation decedents, 1151 cardiovascular deaths occurred (including 630 coronary deaths). The odds of cardiovascular death increased linearly with decreasing age of hypertension onset $(P<0.001$ for trend). Compared with non-hypertensive participants,

\section{WHAT IS ALREADY KNOWN ON THIS TOPIC}

Data to guide clinicians on the possible importance of distinguishing between hypertension that develops earlier in life versus later in life are currently scant

\section{WHAT THIS STUDY ADDS}

Early onset compared with late onset hypertension was associated with greater odds of cardiovascular death and with greater risk for hypertension in offspring Our findings suggest that age of hypertension onset is a trait that both reflects a heritable predisposition for raised blood pressure and offers important prognostic information in assessing an individual's cardiovascular risk hypertension onset at age $<45$ years conferred an odds ratios of 2.2 (1.8 to 2.7) for cardiovascular death and 2.3 (1.8 to 2.9) for coronary death, whereas hypertension onset at age $\geq 65$ years conferred a lower magnitude odds ratios of 1.5 (1.2 to 1.9) for cardiovascular death and 1.4 (0.98 to 1.9) for coronary death $(P \leq 0.002$ for differences in odds ratios between hypertension onset at age $<45$ and age $\geq 65$ ).

\section{CONCLUSIONS}

Early onset and not late onset hypertension in parents was strongly associated with hypertension in offspring. In turn, early onset compared with late onset hypertension was associated with greater odds of cardiovascular, and particularly coronary, death. These findings suggest it may be important to distinguish between early onset and late onset hypertension as a familial trait when assessing an individual's risk for hypertension, and as a specific type of blood pressure trait when estimating risk for cardiovascular outcomes in adults with established hypertension.

\section{Introduction}

Hypertension remains a major risk factor for cardiovascular morbidity and mortality worldwide. ${ }^{1}$ Growing evidence points to the potential benefit of treating hypertension with blood pressure goals approaching the normotensive range. ${ }^{23}$ As clinical practice shifts further towards the goal of maintaining an ideal blood pressure, there is now also increasing emphasis on primordial prevention and identifying those at risk for developing hypertension. ${ }^{4-6}$ Although it is well recognized that the majority of "essential" (ie, primary) hypertension results from environmental exposures superimposed on an inherited predisposition, the extent to which genetic versus lifestyle factors and their interactions determine risk of hypertension remains unclear. $^{78}$ Given that hypertension is a heterogeneous as well as complex trait, it is conceivable that the less common forms of hypertension that tend to manifest earlier in life are more genetically determined, whereas the more common forms that tend to present later in life are more environmentally determined. This notion may motivate the extent to which individuals presenting with hypertension in the clinical setting are screened for inherited factors or initially treated with antihypertensive drugs. ${ }^{910}$

It is well known that hypertension confers substantial risk for developing cardiovascular outcomes when present in either younger or older age. However, there are currently scant data to guide clinicians on the possible relevance of distinguishing between the 
importance of hypertension that develops earlier in life and that which develops later in life. Early onset compared with late onset hypertension is not only more likely to be a heritable risk trait but is perhaps also associated with greater risk for adverse outcomes, although the latter premise has yet to be demonstrated. To test these hypotheses, we conducted an investigation in the family based Framingham Heart Study to assess the extent to which early onset versus late onset hypertension represents a trait inherited from parents and, in turn, the long term risks associated with developing early onset versus late onset hypertension over the adult life course. For this study, we used objectively ascertained blood pressure measurements collected from serial examinations attended by two generations of Framingham participants over a span of six decades, along with concurrent surveillance data on disease outcomes.

\section{Methods \\ Cohorts}

The first generation (“original”) cohort of the Framingham Heart Study included 5209 respondents of a random sample of two thirds of the adult population of Framingham, Massachusetts, who were enrolled in a longitudinal community based cohort study, wherein they participated in biennial examinations beginning in 1948. The second generation (“offspring”) cohort includes 5124 participants of the first generation cohort and their spouses, who were re-examined eight years after the first examination in 1971 and then approximately every four years thereafter. The characteristics and study protocol of both cohorts have been published elsewhere. ${ }^{11} 12$ Each participant provided written informed consent.

\section{Clinical evaluation and definitions}

At each study cycle, participants in both cohorts provided a medical history, including information on medication use, and underwent a physical examination and laboratory assessment of cardiovascular risk factors. ${ }^{11-13}$ Conventional risk factors were measured at all examinations in the offspring cohort whereas smoking status, serum total cholesterol level, and blood glucose level were determined at 25,19 , and 22 out of 28 examinations of the original cohort, respectively. At all examinations, a physician performed two sequential measurements of blood pressure using a mercury column sphygmomanometer on the left arm of seated participants according to a standardized protocol. We defined the blood pressure at a given examination as the mean of the two sequentially measured blood pressure values from that clinical assessment. For participants in both cohorts, given the availability of blood pressure data collected from multiple serial examinations, we defined hypertension as blood pressure $\geq 140 / 90 \mathrm{~mm} \mathrm{Hg}$ or use of antihypertensive drugs on two or more consecutively attended examinations. We selected this definition to reduce variation based on only one measurement and to represent a durable change in blood pressure. Consistent with the definition of hypertension and onset of disease reported in multiple previous studies, we also defined onset of hypertension as the first examination at which criteria for hypertension were met. ${ }^{14-16}$

Assessment of early onset hypertension was based on all available blood pressure measurements performed at serial examinations attended by the participants in the original cohort from 1948 through 2005 and participants in the offspring cohort from 1971 through 2008. We defined early onset hypertension as being present (ie, binary variable) if hypertension was objectively ascertained in an individual who was aged $<55$ years at the time of blood pressure measurement, in accordance with use of this same age threshold in previous studies of hypertension and in the hypertension guidelines of the National Institute for Health and Care Excellence. ${ }^{141718}$ Although information on the use of antihypertensive drugs was not available for the three initial examinations of participants in the original cohort (1948-56), we considered these participants as having no antihypertensive drug use given that blood pressure treatment thresholds during that period were high (180200/100-110 mm Hg) and the efficacy of available antihypertensive drugs during this same period was limited. ${ }^{19}$

\section{Onset of hypertension in parents and risk of hypertension in offspring}

To study the effects of early onset versus late onset hypertension in parents on the risk of hypertension in their offspring, we focused our analysis on participants of the Framingham offspring cohort who had both parents in the original cohort $(n=2470)$ with directly measured blood pressure data available. From this sample we excluded offspring who had hypertension at baseline $(n=461)$, did not attend follow-up examinations $(n=159)$, or had missing covariates $(n=44)$. We also excluded offspring if the age of hypertension onset in parents could not be determined-for example, if one parent or both parents aged $\geq 55$ years had hypertension at their baseline examination $(n=130)$ or if one or both parents did not attend any follow-up examinations $(\mathrm{n}=41)$. Thus, the final sample for this analysis was 1635 .

To capture a relatively specific measure of risk exposure, we categorized parental hypertension status as having both parents free of hypertension; one or both parents with late onset hypertension; at least one parent with early onset hypertension (including only one parent with early onset hypertension, or one parent with early onset and one parent with late onset hypertension); or both parents with early onset hypertension. We compared baseline characteristics between the offspring groups using one way analysis of variance for continuous variables and $\chi^{2}$ tests for categorical variables. We calculated hypertension incidence rates per 1000 person years and estimated 95\% confidence intervals using a Poisson distribution. We compared the cumulative incidence curves of hypertension between groups using Kaplan-Meier analysis and the log-rank test. Because dates of hypertension onset were grouped, we defined the date of hypertension onset in offspring 
participants as the median date of each examination cycle, and we used a discrete time logistic model to assess the association between parental age of hypertension onset and risk of hypertension in offspring participants. We adjusted for age, sex, body mass index, smoking status, systolic blood pressure, diastolic blood pressure, total cholesterol level, and high density lipoprotein cholesterol level. We did not include diabetes status as a covariate in the main models given its extremely low prevalence at baseline: 5 of 1635 participants $(0.3 \%)$. We assessed the heterogeneity of the effect of parental hypertension status between men and women using a multiplicative interaction term. We estimated robust standard errors using a sandwich covariance matrix that incorporates estimates of correlation between offspring. ${ }^{20}$ Given the long duration of follow-up, we investigated how younger compared with older age at risk in offspring participants (ie, age during follow-up or, alternatively, attained age) might influence the association between parental hypertension status and incident hypertension in offspring participants. Specifically, we repeated analyses using offspring cohort follow-up data, divided into two age at risk bands ( $<55$ and $\geq 55$ years). We then estimated age at risk specific hazard ratios by incorporating an interaction term between age at risk and parental hypertension status in models with adjustment for the same previously mentioned covariates except for age, which was replaced by year of birth. ${ }^{21}$ In secondary analyses, we examined the extent to which age of parents at hypertension onset was associated with onset age in offspring, in those offspring participants who developed hypertension $(n=463)$ and had one hypertensive parent or two. For these analyses, we used multivariable adjusted regression models fit with generalized estimating equations (to account for inter-sibling correlations) and defined parental age at onset of hypertension as the lower of the two available ages if both parents developed hypertension. We also performed a sensitivity analysis, with early onset hypertension defined as onset age $<45$ years, instead of $<55$ years, after excluding those with at least one parent aged $\geq 45$ years with hypertension at their baseline examination.

\section{Age of hypertension onset and cause specific mortality}

Because the date of hypertension onset was not available at baseline, we investigated the relation between age of hypertension onset (categorized into five age groups) and cause specific mortality in the original cohort using a case-control design. We focused on the original cohort for this analysis, given the extended duration of follow-up and the relative scarcity of potentially important confounders (ie, use of antihypertensive drugs) during the first 40 years of surveillance for this cohort. From the 5209 first generation participants, we excluded those who had not died before 31 December 2013 ( $n=100)$, did not attend any follow-up examinations ( $n=134)$, had died at age $<45$ years $(n=23)$, had missing covariates $(n=154)$, or had an age at hypertension onset that could not be determined (eg, participant was hypertensive at baseline and aged $\geq 45$ years, $n=1184$ ). Thus, the final sample was 3614 for this analysis.

We used a validated cause specific mortality endpoint for outcomes analyses, ${ }^{22} 23$ which allowed for the exposure variable to consistently precede the outcome variable in timing. Given the extended follow-up duration of the original cohort, with few participants still alive by the last follow-up date, a cause specific mortality approach allowed for almost all individuals to contribute to the outcomes analysis as either a case (ie, death due to cardiovascular disease) or control (ie, death not due to cardiovascular disease). We categorized decedents into five categories based on their age at hypertension onset, with those who died without ever developing hypertension serving as the referent group. In case-control comparisons, we related the age at hypertension onset to cause specific death: cases were considered those who died from cardiovascular disease $(n=1151)$ or those who died specifically from coronary heart disease $(n=630)$, and controls included the remaining 2463 decedents. Owing to the relatively low number of participants who had died specifically from stroke ( $n=221)$, we examined stroke deaths separately in a supplementary analysis. Criteria for adjudication of cardiovascular, coronary, and stroke deaths have been described previously. ${ }^{24}$ We used logistic regression to calculate odds ratios for cases versus controls, adjusted for age at death, sex, smoking status, total cholesterol level, and diabetes (defined as non-fasting plasma glucose level $\geq 11.1 \mathrm{mmol} / \mathrm{L}$ or treatment with antidiabetic drugs). We drew the modifiable covariates (smoking, cholesterol, and diabetes) from first examination cycle at which data were available. We assessed model goodness-of-fit using the Akaike information criterion. We also performed sensitivity analyses with modifiable covariates drawn from the examination at which the age of the decedent was closest to the mean age of hypertension onset in the sample (55.8 years) where data were available. We tested for a linear trend in odds ratios across categories of age at hypertension onset.

All analyses were performed with SAS software version 9.4 (SAS Institute, Cary, NC). We considered a two sided $\mathrm{P}<0.05$ to be statistically significant.

\section{Patient involvement}

No participants were involved in developing research questions or outcome measures, nor were they involved in developing plans for design, recruitment, or implementation of the study. No participants were asked to advise on data interpretation or drafting of the manuscript. Research findings from the Framingham Heart Study are reported on the study website (www.framinghamheartstudy.org/) and disseminated to participants as part of mailed newsletters annually.

\section{Results}

Table 1 shows the baseline characteristics of the 1635 participants in the offspring cohort by categories defined according to parental age of hypertension onset. The groups differed slightly in terms of age, 


\begin{tabular}{|c|c|c|c|c|c|}
\hline Characteristics & \multicolumn{4}{|c|}{ Parental hypertension status } & Pvalue \\
\hline No of examinations & $6.5(1.7)$ & $6.7(1.8)$ & $6.6(1.9)$ & $6.7(1.9)$ & 0.47 \\
\hline Age (years) & $33.3(9.8)$ & $32.9(9.6)$ & $31.6(8.3)$ & $31.6(8.3)$ & 0.03 \\
\hline Body mass index & $23.5(3.3)$ & $24.0(3.7)$ & $24.1(3.6)$ & $24.4(4.1)$ & 0.10 \\
\hline No (\%) current smoker & $58(54.2)$ & $195(40.6)$ & $306(42.4)$ & $158(48.3)$ & 0.02 \\
\hline Cholesterol (mmol/L) & $5.1(0.9)$ & $4.9(0.9)$ & $4.9(1.0)$ & $5.0(0.9)$ & 0.049 \\
\hline High density lipoprotein cholesterol (mmol/L) & $1.3(0.4)$ & $1.3(0.4)$ & $1.4(0.4)$ & $1.3(0.4)$ & 0.50 \\
\hline Systolic blood pressure $(\mathrm{mm} \mathrm{Hg})$ & $114(10)$ & $115(11)$ & $117(10)$ & $119(11)$ & $<0.001$ \\
\hline
\end{tabular}

*Including one parent with early onset hypertension only and one parent with early onset plus one with late onset hypertension.

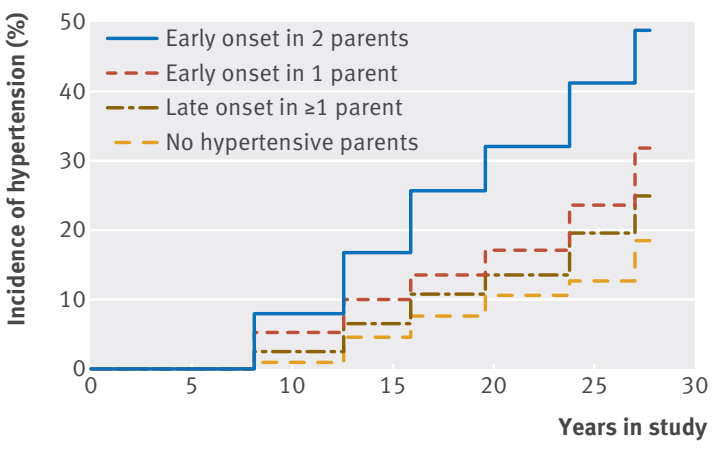

Fig 1 | Cumulative incidence of hypertension in relation to parental age at onset of hypertension

smoking status, and serum total cholesterol level, and, more noticeably, in terms of systolic and diastolic blood pressure.

\section{Hypertension onset age in parents and} hypertension risk in offspring

Over a mean follow-up of 26 (SD 9) years, 481 of 1635 offspring participants (29.4\%) developed hypertension. A progressive increase in hypertension was seen across all categories during follow-up. This increase was the greatest in those whose parents both had early onset hypertension and the lowest in those with non-hypertensive parents (fig 1 , log-rank $\mathrm{P}<0.001$ ). The incidence of hypertension was nearly three times greater in those with parents who both had early onset hypertension than in those with non-hypertensive parents (table 2). In the subgroup of 1488 offspring participants who were aged $<45$ years at baseline, the age specific incidence of hypertension at 45, 55, 65, and 75 years increased across the four groups according to parental hypertension status (see supplementary figure 1).

In unadjusted analyses, we observed that having a parent with late onset hypertension did not significantly increase the risk of hypertension compared with having normotensive parents; by contrast (table 2), having one parent with early onset hypertension was related to a hazard ratio of 1.93 (95\% confidence interval 1.13 to 3.29; $\mathrm{P}=0.02$ ) for hypertension. Having two parents with early onset hypertension was associated with a hazard ratio of 3.56 (2.06 to 6.16; $\mathrm{P}<0.001$ ) for hypertension. Adjustment for covariates did not materially alter these results. We investigated how age at risk affects the association between incident hypertension and parental hypertension status by dividing the follow-up into two age at risk bands ( $<55$ and $\geq 55$ years). Parental hypertension status carried a similar risk for incident hypertension in both age at risk bands (see supplementary table 1). We observed no heterogeneity of the effect of parental hypertension status between men and women when we added a sex interaction term in the adjusted models $(\mathrm{P}=0.68)$.

After excluding participants with at least one parent aged $\geq 45$ years with hypertension at their baseline examination, we performed a sensitivity analysis in

Table 2 | Incident hypertension in offspring according to age of hypertension onset in parents

\begin{tabular}{|c|c|c|c|c|}
\hline \multirow[b]{2}{*}{ Characteristics } & \multicolumn{4}{|c|}{ Parental hypertension status } \\
\hline & $\begin{array}{l}\text { No hypertension } \\
(n=107)\end{array}$ & $\begin{array}{l}\text { Late onset in } \geq 1 \\
\text { parents }(n=480)\end{array}$ & $\begin{array}{l}\text { Early onset in } \\
1 \text { parent }(n=721) t\end{array}$ & $\begin{array}{l}\text { Early onset in both } \\
\text { parents }(n=327)\end{array}$ \\
\hline No of person years & 3052 & 13071 & 18888 & 7813 \\
\hline No with incident hypertension & 18 & 108 & 207 & 148 \\
\hline Incidence per 1000 person years $(95 \% \mathrm{Cl})$ & 5.9 (3.7 to 9.4) & $8.3(6.8$ to 10.0$)$ & $11.0(9.6$ to 12.6$)$ & 18.9 (16.1 to 22.3$)$ \\
\hline \multicolumn{5}{|l|}{ Hazard ratio $(95 \% \mathrm{Cl})$} \\
\hline Unadjusted model & 1.00 (reference) & $1.43(0.82$ to 2.50$)$ & 1.93 (1.13 to 3.29)‡ & $3.56(2.06$ to 6.16$) \S$ \\
\hline Multivariable adjusted model ${ }^{\star}$ & 1.00 (reference) & 1.50 (0.85 to 2.68) & 2.04 (1.18 to 3.54)‡ & $3.45(1.93$ to 6.14$) \S$ \\
\hline \multicolumn{5}{|c|}{ 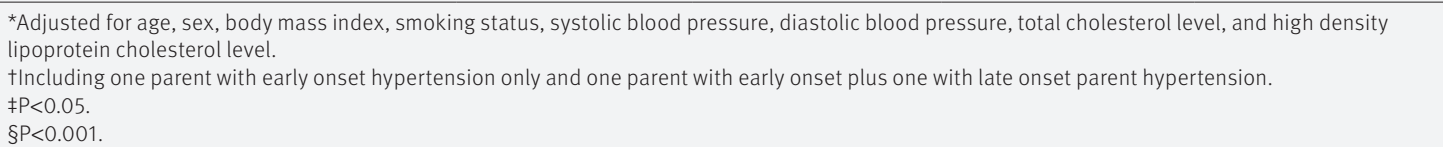 } \\
\hline
\end{tabular}


1297 offspring participants with early onset hypertension, defined as onset age $<45$ years instead of $<55$ years (see supplementary table 2 ); the results of these analyses were similar to those of the main models (table 2). In secondary analyses examining the relation of age at onset of hypertension between parents and offspring, a 10 year lower onset age in parents was associated with a 0.71 year ( $95 \%$ confidence interval 0.13 to 1.28; $\mathrm{P}=0.02$ ) lower onset age in offspring.

\section{Hypertension onset age and risk for cardiovascular death}

Table 3 shows the characteristics of the deceased participants in the original cohort by case-control status. Certain demographic and clinical characteristics were expectedly more prominent among those who had died of cardiovascular causes versus non-cardiovascular

\begin{tabular}{|c|c|c|c|}
\hline \multirow[b]{2}{*}{ Characteristics } & \multicolumn{3}{|l|}{ Cause of death } \\
\hline & $\begin{array}{l}\text { All } \\
\text { cardiovascular } \\
\text { disease }(n=1151)\end{array}$ & $\begin{array}{l}\text { Coronary } \\
\text { heart disease } \\
(n=630)\end{array}$ & $\begin{array}{l}\text { Non- } \\
\text { cardiovascular } \\
\text { disease }(n=2463)\end{array}$ \\
\hline No of examinations & $15.1(6.7)$ & $14.1(6.0)$ & $16.3(7.0)$ \\
\hline Age at death (years) & $77.1(11.8)$ & $74.2(11.4)$ & $80.9(12.0)$ \\
\hline No (\%) of women & $541(47.0)$ & $229(36.4)$ & $1429(58.0)$ \\
\hline \multicolumn{4}{|l|}{ Age of hypertension onset (years): } \\
\hline$<45$ & $295(25.6)$ & $185(29.4)$ & $391(15.9)$ \\
\hline $46-54$ & $211(18.3)$ & $120(19.1)$ & $343(13.9)$ \\
\hline $55-64$ & 198 (17.2) & $90(14.3)$ & $400(16.2)$ \\
\hline$\geq 65$ & $185(16.1)$ & $79(12.5)$ & $526(21.4)$ \\
\hline No (\%) with no hypertension & $262(22.8)$ & $156(24.8)$ & $803(32.6)$ \\
\hline No (\%) current smoker & $697(60.6)$ & $397(63.0)$ & $1459(59.2)$ \\
\hline Cholesterol $(\mathrm{mmol} / \mathrm{L})^{\star}$ & $6.4(1.2)$ & $6.0(1.2)$ & $5.6(1.1)$ \\
\hline No (\%) with diabetes mellitus* & $15(1.3)$ & $8(1.3)$ & $10(0.4)$ \\
\hline
\end{tabular}

*At first examination cycle where data were available.

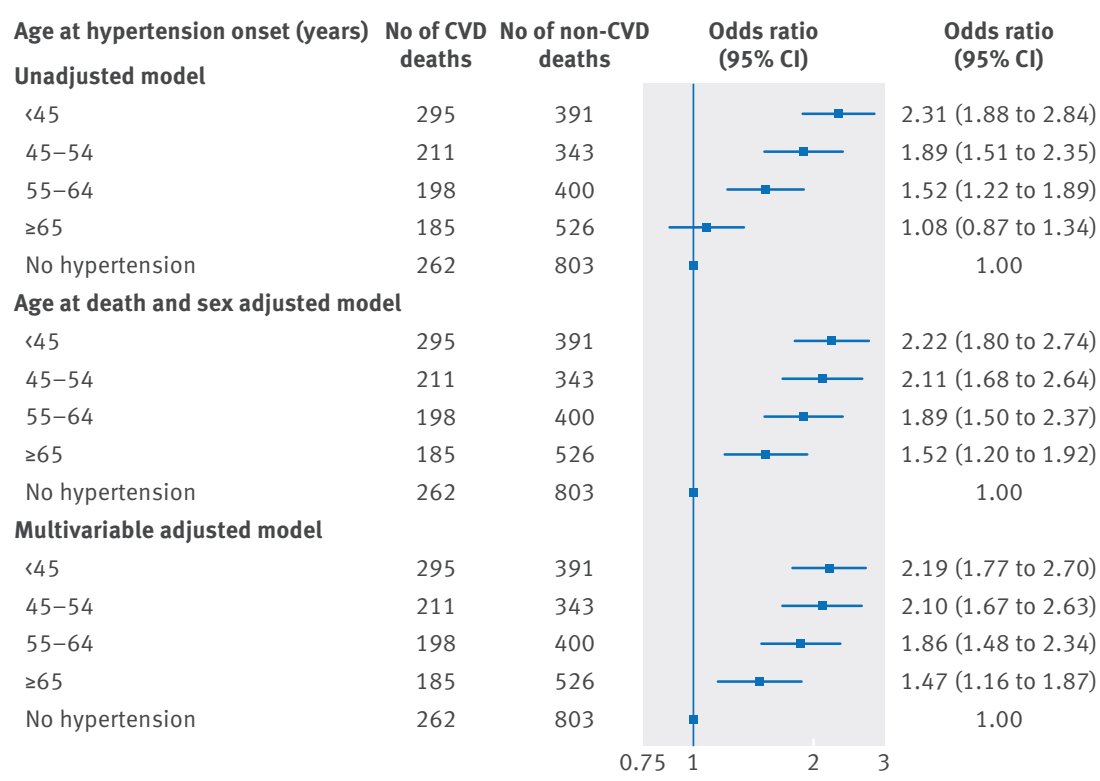

Fig 2 | Odds of cardiovascular death versus non-cardiovascular death by age at onset of hypertension. Age at death, sex, smoking status, serum total cholesterol level, and diabetes are included as covariates in the multivariable adjusted model. $\mathrm{CVD}=$ cardiovascular disease causes. Of note, participants who had died of cardiovascular and coronary heart disease had experienced hypertension onset at a younger age than those who died of non-cardiovascular causes. Figure 2 and supplementary figure 2 display the main results of analyzing risk of cardiovascular versus non-cardiovascular deaths by age group of hypertension onset. The trends of decreasing age of hypertension onset in relation to increasing odds of cardiovascular and coronary mortality were significant in both unadjusted and adjusted analyses $(\mathrm{P}<0.001$ for all).

Compared with participants without hypertension, hypertension onset before age 45 was associated with an odds ratio of 2.19 (95\% confidence interval 1.77 to 2.70; $\mathrm{P}<0.001)$ of cardiovascular death; by contrast, hypertension onset at age $\geq 65$ years was associated with only an odds ratio of 1.47 (1.16 to $1.87 ; \mathrm{P}=0.001$ ) for cardiovascular death (fig 2). Similarly, hypertension onset before age 45 was related to an odds ratio of 2.26 (1.75 to 2.93; $\mathrm{P}<0.001$ ) for coronary death and hypertension onset at age $\geq 65$ years did not confer a significantly greater odds of coronary death (odds ratio 1.36, 95\% confidence interval 0.98 to $1.87 ; \mathrm{P}=0.07$; see supplementary figure 2). Furthermore, the odds of cardiovascular death $(1.48,1.16$ to $1.89 ; \mathrm{P}=0.002)$ and coronary death (1.67, 1.21 to $2.31 ; \mathrm{P}=0.002)$ were significantly higher in participants with hypertension onset before age 45 than in those with onset at age $\geq 65$ years (fig 2 ). We observed a trend in increasing odds of stroke death with decreasing age of hypertension onset $(\mathrm{P}<0.001$ for trend, see supplementary table 3); this trend was significant albeit less evident than the trends observed for cardiovascular death and coronary death, given the relatively lower number of stroke deaths. In the model for cardiovascular death (fig 2), we observed improvement in model goodness of fit, compared with the unadjusted model (Akaike information criterion (AIC) 4447), after adding sex and age at death as covariates (AIC=4361). We observed a more modest further improvement in model fit after additionally including the other clinical covariates (AIC=4322). In additional analyses that included weight as a covariate in the models, results were not substantially changed (data not shown). In sensitivity analyses including covariates drawn from the examination at which the decedent age was closest to the mean age of hypertension onset of the sample, results were similar (see supplementary figure $3, \mathrm{P}<0.001$ for trend).

\section{Discussion}

The availability of serial, standardized blood pressure measurements performed in the family based Framingham Heart Study provided the unique opportunity to investigate the risks associated with early onset hypertension compared with late onset hypertension in the community. We observed that early and not late onset hypertension in parents predicted incident hypertension in offspring. In turn, the presence of early onset compared with late onset hypertension was associated with a greater risk for cardiovascular death. These findings suggest it may be important to distinguish between early onset and late onset hypertension as a familial 
trait when assessing an individual's risk for hypertension and as a specific type of blood pressure trait when estimating risk for cardiovascular outcomes in individuals with established hypertension.

\section{Strengths and limitations of this study}

Our study has several strengths, including the large community based sample with objective blood pressure measurements and longitudinal data collected across family members. In addition, we examined for incident hypertension in the original cohort over a period of 60 years; this cohort had a high retention rate and less than $1.9 \%$ of the participants (100 of 5209 participants) were alive at the end of follow-up. Thus, our analyses included near complete data for both early onset and late onset hypertension in the parental cohort. Because antihypertensive drug use was relatively uncommon and had limited efficacy during the first four decades of assessments in the original cohort, ${ }^{19}$ results of our outcomes analyses are relatively unconfounded by antihypertensive drug use; thus, potential implications of the outcomes results may be particularly relevant for the large proportion of individuals with hypertension who remain untreated or undertreated worldwide. ${ }^{25}$ However, several limitations of our study also merit consideration. We observed a trend in increasing odds of stroke death with decreasing age of hypertension onset, although this analysis was limited by the smaller fraction of total cardiovascular deaths being attributable to stroke in our study. Thus, the observed point estimates from this analysis were less stable with wider confidence intervals, reflecting limited power and providing suggestive albeit less than definitive results on the relation of age at hypertension onset with stroke death. The follow-up intervals in the original and offspring cohorts of the Framingham Heart Study differed as parents underwent biennial examinations whereas their offspring were re-examined eight years after the first examination and every four years thereafter. However, because the cohorts were not pooled for analyses, this discrepancy in time intervals between examinations most likely did not substantially influence the main results. For some individuals, hypertension may arise secondary to a specific neurohormonal, renal, or other medical condition that not only warrants specific treatment but also confers additional adverse risk. In our study, possible secondary causes of hypertension were not routinely assessed as part of the standardized examination. Although the prevalence of secondary hypertension is reportedly less than $1 \%$ in the community, ${ }^{26}$ it is more likely to be present in earlier rather than later life and so additional studies are needed to examine whether the presence of secondary versus primary hypertension partly accounts for or impacts the association of age at hypertension onset with outcomes. Precise data on physical activity and dietary patterns were not available for the offspring cohort. None the less, these factors are highly correlated with body mass index, which was included in all models, and body mass index likely mediates their effect on blood pressure..$^{27}$ Our study sample included predominantly white adults of European ancestry and thus the generalizability of our results to other racial or ethnic groups remains unknown. Because the outcomes analyses were based on a case-control design, additional studies in prospective as well as more diverse populations are needed to validate our main findings.

\section{Comparison with other studies}

With an increasing clinical focus on maintaining blood pressure values within the normotensive range, ${ }^{22829}$ there is ever growing interest in identifying those at risk for developing hypertension who may benefit from early interventions. ${ }^{4-6}$ Because hypertension is known to result from environmental exposures acting on top of an inherited predisposition, numerous studies have sought to characterize its genetic determinants. Interestingly, genetic risk scores have not been shown to appreciably add to familial history when predicting incident hypertension. ${ }^{30}$ This phenomenon is likely due to the fact that family history captures information on shared lifestyle and dietary patterns ${ }^{31}$ as well as the general tendency for multiple cardiovascular risk factors to cluster within families. Therefore, we examined the extent to which early onset versus late onset hypertension in parents predicts incident hypertension in offspring to leverage the information offered by family history while also refining its potential to improve risk assessment. Our results suggest that a precisely defined family history of early onset hypertension is both a robust and a widely accessible tool for predicting incident hypertension. Although previous studies have shown that any hypertension in parents increases the risk of incident hypertension by 1.3 -fold to 2.0 -fold in children ${ }^{30} 3233$ and adults, ${ }^{34-37}$ previous investigations have all relied on participants' self report for determining parental history of hypertension and thus have been limited by reporting bias and potentially inaccurate estimates of risk. ${ }^{38}$ Interestingly, the risk estimates reported in most published studies are lower than the estimates we observed for objectively assessed early onset hypertension in Framingham. This discrepancy suggests that a portion of the risk conveyed by parental hypertension may have been previously missed owing to lack of precision in capturing parental history of hypertension, or owing to attenuation of effects observed when not distinguishing between early onset versus late onset hypertension as a potentially heritable trait. To our knowledge, only two previous studies have attempted to assess the relative impact of parental early onset versus late onset hypertension on risk for hypertension in offspring, with conflicting results. ${ }^{1418}$ Hunt and colleagues examined participant reported hypertension in relation to a reported family history of hypertension; in the absence of available data on blood pressure measurement for either parents or offspring, analyses relied on a reported age of hypertension diagnosis in parents instead of any objectively assessed age at onset of hypertension. In these analyses, familial early onset and late onset hypertension were similarly associated with risk of hypertension in offspring. ${ }^{18}$ Wang and colleagues also relied on participant reported age of hypertension diagnosis in parents, and related 
this trait with offspring blood pressure that was self measured using a mercury sphygmomanometer by study participants who were all male physicians. ${ }^{14}$ Although the study was based on a selected study sample of health professionals trained at Johns Hopkins, of whom only 18 participants had two parents with reported early onset hypertension, the researchers observed a greater risk of hypertension among male physicians whose parents had early onset compared with late onset hypertension. The present study extends from previous work not only by investigating the age at actual hypertension onset but also by examining its association with cardiovascular risk.

Because hypertension is known to confer substantial risk for a variety of cardiovascular as well as non-cardiovascular diseases, concerted efforts have been devoted to developing more accurate assessments of hypertension related risk among vulnerable individuals. Accordingly, several studies have shown that distinct blood pressure trajectory patterns and antecedent time averaged or cumulative blood pressure ( $\mathrm{mm} \mathrm{Hg \times year)} \mathrm{may} \mathrm{be} \mathrm{more}$ effective than a single visit blood pressure measure in predicting target organ damage and adverse events. ${ }^{39-43}$ While confirming the importance of capturing exposure during the life course, these approaches have limited utility in clinical practice, wherein longitudinal data collected from standardized repeated blood pressure measures are not typically available. Our study findings suggest that age at hypertension onset is an easily ascertained trait that not only reflects an inherited predisposition for raised blood pressure but also offers important prognostic information in assessing an individual's cardiovascular risk. Multiple previous studies have shown that hypertension confers cardiovascular risk whether present in younger age or older age, but prognostic data related to hypertension that manifests earlier in life versus later have been extremely limited. We found a single study of hypertensive primary care patients and normotensive controls, ${ }^{44}$ published in 1987, which reported that the patients who received a hypertension diagnosis during their fifth decade of life had a 5.2-fold odds of developing a cardiovascular event compared with non-hypertensive patients; by contrast, patients who received a diagnosis of hypertension during their seventh decade of life had a corresponding odds ratio of only 1.2. No confidence intervals for the odds ratios were reported. The odds ratios were also unadjusted for age at death and other conventional cardiovascular risk factors, and may therefore only reflect the rising impact of other disease events, such as cancer, with age. None the less, our results are concordant with these historical findings, while based on analyzing objectively measured blood pressure data and more precise estimates of age at hypertension onset, in both parents and offspring.

\section{Conclusions and policy implications}

Our results show that early, and not late, onset hypertension in parents is a strong risk factor for incident hypertension in offspring as well as for cardiovascular death in those with hypertension. Whereas current practice involves only collecting data on family history on the presence or absence of hypertension in parents, our findings suggest the added value of determining age at onset in affected parents. Given their observed increased risk for hypertension, those whose parents had early onset hypertension would likely benefit from blood pressure monitoring at regular intervals and lifestyle counseling. For individuals with already manifest early onset hypertension, our findings indicate a risk for cardiovascular events even greater than in those with late onset hypertension. Several studies have reported that some younger individuals with early onset hypertension may be less likely than older individuals to receive aggressive blood pressure management in practice, ${ }^{254546}$ and our results underscore the need for appropriate care in these at risk individuals and potentially more targeted antihypertensive treatment. Further research is warranted to determine if age at hypertension onset could be used to estimate cardiovascular risk with greater precision and guide treatment. Additional work is also needed to evaluate the extent to which specific interventions may be warranted for preventing or managing early onset versus late onset hypertension.

We thank the participants and staff of the Framingham Heart Study for their valuable contributions.

Contributors: TJN, SC, and ELM designed the study. All authors were involved in analyzing and interpreting the data. TJN and SC drafted the manuscript. ELM, NKL, MGL, MH, and RSV critically revised the manuscript for important intellectual content. TJN, ELM, MGL, and MH performed the statistical analysis. RSV and SC obtained funding and provided administrative support. MGL, RSV, and SC supervised the study. TN and SC are the guarantors.

Funding: This work was supported by the American Heart Association (SC) and the National Heart, Lung and Blood Institute's Framingham Heart Study (contracts N01HC25195 and HHSN268201500001I), and the following National Institutes of Health grants: T32GM74905 (ELM), R01HL093328 (RSV), R01HL107385 (RSV), R01HL126136 (RSV), R00HL107642 (SC), R01HL131532 (SC), and R01HL134168 (SC). The funders played no role in the design of the study; the collection, analysis, and interpretation of the data; and the decision to approve publication of the finished manuscript. All authors had full access to all of the data (including statistical reports and tables) in the study and can take responsibility for the integrity of the data and the accuracy of the data analysis.

Competing interests: All authors have completed the ICMJE uniform disclosure form at www.icmje.org/coi_disclosure.pdf and declare: no support from any organization for the submitted work; no financial relationships with any organizations that might have an interest in the submitted work in the previous three years; no other relationships or activities that could appear to have influenced the submitted work.

Ethical approval: Boston University Medical Center's institutional review board approved all study protocols.

Data sharing: Participant level data are available at the database of Genotypes and Phenotypes (https://www.ncbi.nlm.nih.gov/gap/) Consent for data sharing was not obtained from all participants but the presented data are anonymized and risk of identification is low.

Transparency: The lead authors (TN and SC) affirm that the manuscript is an honest, accurate, and transparent account of the study being reported; that no important aspects of the study have been omitted; and that any discrepancies from the study as planned (and, if relevant, registered) have been explained.

This is an Open Access article distributed in accordance with the Creative Commons Attribution Non Commercial (CC BY-NC 4.0) license, which permits others to distribute, remix, adapt, build upon this work non-commercially, and license their derivative works on different terms, provided the original work is properly cited and the use is noncommercial. See: http://creativecommons.org/licenses/by-nc/4.0/.

\footnotetext{
Lim SS, Vos T, Flaxman AD et al. A comparative risk assessment of burden of disease and injury attributable to 67 risk factors and risk factor clusters in 21 regions, 1990-2010: a systematic analysis for the Global Burden of Disease Study 2010. Lancet 2012;380:222460. doi:10.1016/S0140-6736(12)61766-8.
} 
2 Wright JT Jr,, Williamson JD, Whelton PK, et al. SPRINT Research Group. A Randomized Trial of Intensive versus Standard Blood-Pressure Control. N Engl/ Med 2015:373:2103-16. doi:10.1056/ NEJMoa1511939.

3 Xie X, Atkins E, Lv J, et al. Effects of intensive blood pressure lowering on cardiovascular and renal outcomes: updated systematic review and meta-analysis. Lancet 2016;387:435-43. doi:10.1016/ S0140-6736(15)00805-3

4 Niinikoski H, Jula A, Viikari J, et al. Blood pressure is lower in children and adolescents with a low-saturated-fat diet since infancy: the Special Turku Coronary Risk Factor Intervention Project. Hypertension 2009;53:918-24. doi:10.1161/HYPERTENSIONAHA.109.130146.

5 Laitinen TT, Pahkala K, Magnussen CG, et al. Ideal cardiovascular health in childhood and cardiometabolic outcomes in adulthood: the Cardiovascular Risk in Young Finns Study. Circulation 2012;125:1971 8. doi:10.1161/CIRCULATIONAHA.111.073585.

6 Vasan RS, Beiser A, Seshadri S, et al. Residual lifetime risk for developing hypertension in middle-aged women and men: The Framingham Heart Study. JAMA 2002;287:1003-10. doi:10.1001/ jama.287.8.1003.

7 Zhao Q, Kelly TN, Li C, He J. Progress and future aspects in genetics of human hypertension. Curr Hypertens Rep 2013;15:676-86. doi:10.1007/s11906-013-0388-6.

8 Ehret GB, Caulfield MJ. Genes for blood pressure: an opportunity to understand hypertension. Eur Heart J 2013;34:951-61. doi:10.1093/ eurheartj/ehs455.

9 Mancia G, Fagard R, Narkiewicz K, et al. 2013 ESH/ESC guidelines for the management of arterial hypertension: the Task Force for the Management of Arterial Hypertension of the European Society of Hypertension (ESH) and of the European Society of Cardiology (ESC). Eur Heart / 2013;34:2159-219. doi:10.1093/eurheartj/eht151.

10 Viera AJ, Neutze DM. Diagnosis of secondary hypertension: an age-based approach. Am Fam Physician 2010;82:1471-8.

11 Kannel WB, Feinleib M, McNamara PM, Garrison RJ, Castelli WP. An investigation of coronary heart disease in families. The Framingham offspring study. Am J Epidemiol 1979;110:281-90. doi:10.1093/ oxfordjournals.aje.a112813.

12 Dawber TR, Meadors GF, Moore FE Jr. Epidemiological approaches to heart disease: the Framingham Study. Am J Public Health Nations Health 1951;41:279-81. doi:10.2105/AJPH.41.3.279.

13 Splansky GL, Corey D, Yang Q, et al. The Third Generation Cohort of the National Heart, Lung, and Blood Institute's Framingham Heart Study: design, recruitment, and initial examination. Am J Epidemiol 2007:165:1328-35. doi:10.1093/aje/kwm021.

14 Wang NY, Young JH, Meoni LA, Ford DE, Erlinger TP, Klag MJ. Blood pressure change and risk of hypertension associated with parental hypertension: the Johns Hopkins Precursors Study. Arch Intern Med 2008:168:643-8. doi:10.1001/archinte.168.6.643.

15 Toren P, Margel D, Kulkarni G, Finelli A, Zlotta A, Fleshner N. Effect of dutasteride on clinical progression of benign prostatic hyperplasia in asymptomatic men with enlarged prostate: a post hoc analysis of the REDUCE study. BMJ 2013;346:f2109. doi:10.1136/bmi.f2109.

16 Meigs JB, Cupples LA, Wilson PW. Parental transmission of type 2 diabetes: the Framingham Offspring Study. Diabetes 2000;49:2201-7. doi:10.2337/diabetes.49.12.2201.

17 Krause T, Lovibond K, Caulfield M, McCormack T, Williams B. Guideline Development Group. Management of hypertension: summary of NICE guidance. BM/ 2011;343:d4891. doi:10.1136/bmj.d4891.

18 Hunt SC, Williams RR, Barlow GK. A comparison of positive family history definitions for defining risk of future disease. / Chronic Dis 1986;39:809-21. doi:10.1016/0021-9681(86)90083-4

19 Moser M.Historical perspectives on the management of hypertension. J Clin Hypertens (Greenwich) 2006;8:15-20.

20 Lin DY. Cox regression analysis of multivariate failure time data: the marginal approach. Stat Med 1994;13:2233-47. doi:10.1002/ sim.4780132105

21 Lewington S, Clarke R, Qizilbash N, Peto R, Collins R. Prospective Studies Collaboration. Age-specific relevance of usual blood pressure to vascular mortality: a meta-analysis of individual data for one million adults in 61 prospective studies. Lancet 2002;360:1903-13. doi:10.1016/S0140-6736(02)11911-8

22 Zaridze D, Lewington S, Boroda A, et al. Alcohol and mortality in Russia: prospective observational study of 151,000 adults. Lancet 2014;383:1465-73. doi:10.1016/S0140-6736(13)62247-3.

23 Alegre-Díaz J, Herrington W, López-Cervantes M, et al. Diabetes and Cause-Specific Mortality in Mexico City. N Engl J Med 2016;375:196171. doi:10.1056/NEJMoa1605368

24 Kannel WB, Wolf PA, Garrison RJ, eds. Some risk factors related to the annual incidence of cardiovascular disease and death in pooled repeated biennial measurements. Framingham Heart Study, 30 Year Follow-Up.US Department of Health and Human Services, 1987.

25 Chow CK, Teo KK, Rangarajan S, et al. PURE (Prospective Urban Rural Epidemiology) Study investigators. Prevalence, awareness, treatment, and control of hypertension in rural and urban communities in high-, middle-, and low-income countries. JAMA 2013;310:959-68. doi:10.1001/jama.2013.184182.
26 Sigurdsson JA, Bengtsson C, Tibblin E, Wojciechowski J. Prevalence of secondary hypertension in a population sample of Swedish women. Eur Heart/1983:4:424-33. doi:10.1093/oxfordjournals.eurheartj.a061489.

27 Hu G, Barengo NC, Tuomilehto J, Lakka TA, Nissinen A, Jousilahti P. Relationship of physical activity and body mass index to the risk of hypertension: a prospective study in Finland. Hypertension 2004:43:25-30. doi:10.1161/01.HYP.0000107400.72456.19.

28 Julius S, Nesbitt SD, Egan BM, et al. Trial of Preventing Hypertension (TROPHY) Study Investigators. Feasibility of treating prehypertension with an angiotensin-receptor blocker. N Engl / Med 2006:354:168597. doi:10.1056/NEJMoa060838.

29 Weber MA, Schiffrin EL, White WB, et al. Clinical practice guidelines for the management of hypertension in the community a statement by the American Society of Hypertension and the International Society of Hypertension. J Hypertens 2014;32:3-15. doi:10.1097/ HJH.0000000000000065.

30 Juhola J, Oikonen M, Magnussen CG, et al. Childhood physical, environmental, and genetic predictors of adult hypertension: the cardiovascular risk in young Finns study. Circulation 2012;126:402-9. doi:10.1161/CIRCULATIONAHA.111.085977.

31 Mitchell BD, Rainwater DL, Hsueh WC, Kennedy AJ, Stern MP, Maccluer JW. Familial aggregation of nutrient intake and physical activity: results from the San Antonio Family Heart Study. Ann Epidemiol 2003:13:128-35. doi:10.1016/S1047-2797(02)00255-7.

32 Shear CL, Burke GL, Freedman DS, Berenson GS. Value of childhood blood pressure measurements and family history in predicting future blood pressure status: results from 8 years of follow-up in the Bogalusa Heart Study. Pediatrics 1986;77:862-9.

33 Burke V, Gracey MP, Beilin LJ, Milligan RA. Family history as a predicto of blood pressure in a longitudinal study of Australian children. J Hypertens 1998;16:269-76. doi:10.1097/00004872-199816030-00003

34 Friedman GD, Selby JV, Quesenberry CP Jr,, Armstrong MA, Klatsky AL. Precursors of essential hypertension: body weight, alcohol and salt use, and parental history of hypertension. Prev Med 1988;17:387-402 doi:10.1016/0091-7435(88)90038-2.

35 Shook RP, Lee DC, Sui X, et al. Cardiorespiratory fitness reduces the risk of incident hypertension associated with a parental history of hypertension. Hypertension 2012;59:1220-4. doi:10.1161/ HYPERTENSIONAHA.112.191676.

36 Fava C, Sjögren M, Montagnana M, et al. Prediction of blood pressure changes over time and incidence of hypertension by a genetic risk score in Swedes. Hypertension 2013;61:319-26. doi:10.1161/ HYPERTENSIONAHA.112.202655.

37 Fava C, Sjögren M, Olsson S, et al. A genetic risk score for hypertension associates with the risk of ischemic stroke in a Swedish case-control study. Eur J Hum Genet 2015;23:969-74. doi:10.1038/ejhg.2014.212.

38 Bensen JT, Liese AD, Rushing JT, et al. Accuracy of proband reported family history: the NHLBI Family Heart Study (FHS). Genet Epidemiol 1999;17:141-50. doi:10.1002 (SICI)1098-2272(1999)17:2<141\%:AID-GEPI4>3.0.CO·2-0.

39 Vasan RS, Massaro JM, Wilson PW, et al. Framingham Heart Study. Antecedent blood pressure and risk of cardiovascular disease: the Framingham Heart Study. Circulation 2002;105:48-53. doi:10.1161/ hc0102.101774.

40 Lauer MS, Anderson KM, Levy D. Influence of contemporary versus 30-year blood pressure levels on left ventricular mass and geometry: the Framingham Heart Study. J Am Coll Cardiol 1991;18:1287-94. doi:10.1016/0735-1097(91)90549-0

41 Wilson PW, Hoeg JM, D’Agostino RB, et al. Cumulative effects of high cholesterol levels, high blood pressure, and cigarette smoking on carotid stenosis. N Engl J Med 1997;337:516-22. doi:10.1056/ NEJM199708213370802

42 Pletcher MJ, Bibbins-Domingo K, Lewis CE, et al. Prehypertension during young adulthood and coronary calcium later in life. Ann Intern Med 2008;149:91-9. doi:10.7326/0003-4819-149-2-200807150-00005.

43 Kishi S, Teixido-Tura G, Ning H, et al. Cumulative Blood Pressure in Early Adulthood and Cardiac Dysfunction in Middle Age: The CARDIA Study. J Am Coll Cardiol 2015;65:2679-87. doi:10.1016/j. jacc.2015.04.042.

44 Buck C, Baker P, Bass M, Donner A. The prognosis of hypertension according to age at onset. Hypertension 1987;9:204-8. doi:10.1161/01.HYP.9.2.204

45 Sivén SS, Niiranen TJ, Aromaa A, Koskinen S, Jula AM. Social, lifestyle and demographic inequalities in hypertension care. Scand J Public Health 2015;43:246-53. doi:10.1177/1403494815571031.

46 Wallace ML, Magnan EM, Thorpe CT, Schumacher JR, Smith MA Johnson HM. Diagnosis and treatment of incident hypertension among patients with diabetes: a U.S. multi-disciplinary group practice observational study. J Gen Intern Med 2015;30:768-76. doi:10.1007/ s11606-015-3202-0.

Supplementary information: supplementary tables 1-3 and figures 1-3 